\title{
Wilson's disease patient with iron metabolism discharge barriers: A case report
}

\author{
GUOEN CAI $^{1}$, XINMING HUANG $^{2}$, QINYONG YE $^{1}$, WENTING XIONG $^{1}$ and QING DUAN ${ }^{2}$ \\ Departments of ${ }^{1}$ Neurology and ${ }^{2}$ Magnetic Resonance Imaging, Fujian Medical University Union Hospital, \\ Fuzhou, Fujian 350001, P.R. China
}

Received September 10, 2015; Accepted October 18, 2016

DOI: $10.3892 /$ etm.2016.3928

\begin{abstract}
Wilson's disease (WD) is an autosomal genetic disease. In the present study, the patient was a 35-year-old woman who exhibited drinking bucking (bulbar paralysis) and dysphagia for a period of nine years. Genetic analysis of the patient identified the Thr935Met and Pro992Leu mutations, which lead to copper metabolism discharge barriers. Moreover, magnetic resonance imaging revealed a susceptibility-weighted imaging (SWI) hyperintense area in the bilateral substantia nigra and lenticular nuclei. These SWI observations indicated that 'mineral deposits' were present. The present case demonstrates that the SWI hyperintense area in the bilateral lenticular nuclei, substantia nigra and red nucleus combined with the patient's symptoms indicated that there is a possibility to diagnose WD when it is not detected by genetic analysis. In addition, it demonstrates that systemic mineral removal treatment (including manganese, iron and copper) may be successful for the initial treatment of WD.
\end{abstract}

\section{Introduction}

Wilson's disease (WD) is an autosomal genetic disease in which the ATP7B gene mutation is localized on chromosome $13(1,2)$. This is known to be the key gene that leads to copper metabolism discharge barriers (1-3). Thus, copper accumulates mainly in the liver, kidney, head and retina and causes abnormal liver function, renal dysfunction and degeneration of the nervous system (4,5). Moreover, widespread copper accumulation in the central nervous system results in cognitive decline, extrapyramidal change and also pyramidal dysfunction (6).

Brain magnetic resonance imaging (MRI) studies of WD patients reveals that the lesions generally appeared to be

Correspondence to: Dr Qinyong Ye, Department of Neurology, Fujian Medical University Union Hospital, 29 Xinquan Road, Fuzhou, Fujian 350001, P.R. China

E-mail: unionqyye@126.com

Key words: Wilson's disease, susceptibility-weighted imaging, compound heterozygous mutation, iron metabolism hyperintense on T2-weighted and hypointense on T1-weighted images (7). It is associated with an increased water content in the brain. Currently available MR techniques, and susceptibility-weighted imaging (SWI) has been demonstrated to be a more sensitive means of detecting mineral deposits (of manganese, iron and copper compared to other conventional MR imaging $(8,9)$. Moreover, the lesions of WD patients mainly include copper deposition. The present study reports a case of newly diagnosed WD in order to characterize the patterns of paramagnetic signal abnormalities by SWI.

\section{Case report}

A case of a 35-year-old woman was admitted to the Fujian Medical University Union Hospital (Fuzhou, China) due to drinking bucking (bulbar paralysis) and dysphagia in March 2012. The patient was diagnosed with WD for nine years, and she had no history of underlying disease.

Furthermore, the patient initially had auditory hallucinations, delusions, disorganized speech and thinking. She was initially diagnosed with schizophrenia in Fuzhou Fourth Hospital (Fuzhou, China) in February 2001 and was the treated for it. However, the patient gradually presented gait disturbance, dysarthria, joint rigid dystonia and risus sardonicus, and she visited Fuzhou Fourth Hospital again in July 2002 where she was diagnosed with WD and was treated with penicillamine (PCA) for three months. However, the patient's neurological symptoms did not improve. Between 2003 and 2005, she had been hospitalized for two months per year and had been treated with PCA (250 mg, four times per day) and zinc (50 mg, three times per day). Furthermore, she had also received Madopar ( $0.125 \mathrm{mg}$, three times per day) and Artane ( $2 \mathrm{mg}$, three times per day) for gait disturbance and dysdipsia aggratate.

Between 2006 and 2008, the patient was treated with PCA, zinc, Madopar and Artane, but between 2009 and 2010, she did not receive any medication. Moreover, the ceruloplasmin levels were found to be normal in 2010. In 2011, the patient experienced symptoms of drinking bucking and visited the Affiliated Union Hospital, where she undertook a brain magnetic resonance imagine (MRI) examination, and her ceruloplasmin levels were measured. Physical examinations showed that she had a temperature of $36.5^{\circ} \mathrm{C}$, heart rate of 80 beats/min and blood pressure of $110 / 80 \mathrm{mmHg}$. 

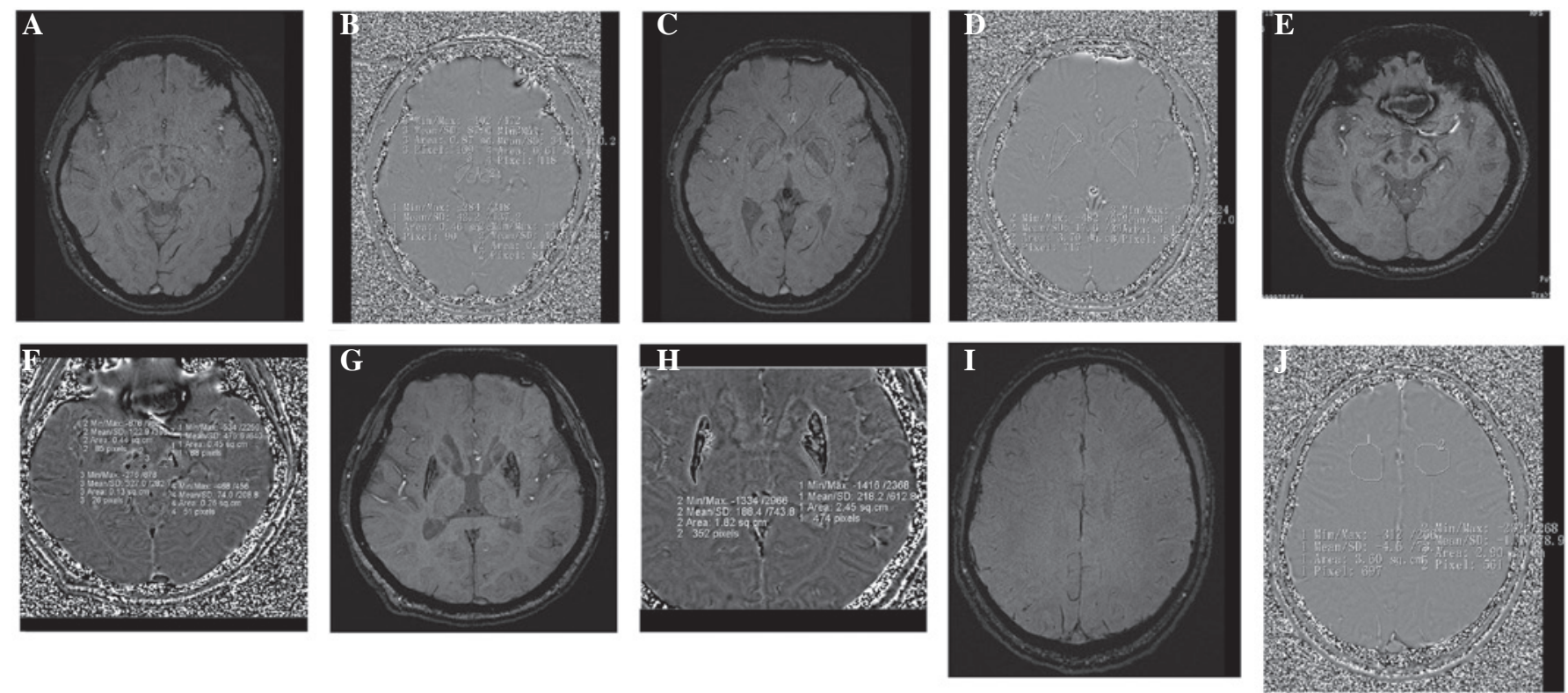

Figure 1. SWI in the lenticular nucleus, substantia nigra, red nucleus and frontal lobe. (A-D) The low signal of iron deposition in (A and B) bilateral substantia nigra and the red nucleus (C and D) and lenticular nuclei are shown in SWI imaging and corrected phase imaging in a healthy patient. (E-H) The high signal intensities of iron deposition in ( $\mathrm{E}$ and $\mathrm{F}$ ) bilateral substantia nigra and the red nucleus $(\mathrm{G}$ and $\mathrm{H})$ and lenticular nuclei are shown in SWI imaging and corrected phase imaging in WD patients. (I and J) I and J show a corrected phase imaging of the frontal lobe white matter region. SWI, susceptibility-weighted imaging; WD, Wilson's disease.

Table I. Laboratory data on admission.

\begin{tabular}{lc}
\hline Characteristic & Value \\
\hline Hemoglobin (g/l) & 128.0 \\
White blood cells (cells/l) & $3.8 \times 10^{9}$ \\
Neutrophils (\%) & 70.1 \\
Blood platelets (platelets/l) & $175 \times 10^{9}$ \\
Ceruloplasmine (g/l) & 0.23 \\
Proteinuria & $1+$ \\
Albuminuria & $(-)$ \\
Fibrinogen (g/l) & 4.23 \\
AST (IU/l) & $<40$ \\
ALT (IU/l) & $<40$ \\
\hline
\end{tabular}

AST, aspartate aminotransferase; ALT, alanine aminotransferase.

Furthermore, the patient presented mild dysarthria and rigid dystonia. The patient's abdomen was also soft but not tender, without organomegaly and no edema was identified. Laboratory results are shown in Table I. The results were negative for the serum antinuclear antibody, hepatitis B surface antigen, hepatitis B core antibody and hepatitis C antibody. In addition, the serum ceruloplasmin level was $<2 \mathrm{mg} / \mathrm{dl}$. An MRI revealed an abnormal hypointensity of the liver on the T2-weighted images. Finally, the patient was diagnosed with WD, and her gene was identified to have a mutation in the C-to-T transition (Thr935Met) and the C-to-A transition (Pro992Leu). Furthermore, an SWI of the brain was captured and high signal intensities were apparent in the bilateral substantia nigra and lenticular nuclei (Fig. 1). Informed consent was obtained from patients.

\section{Discussion}

SWI is used to demonstrate the magnetic susceptibility difference between the magnetic material tissue and non-containing magnetic material tissue $(10,11)$. More specifically, the corrected phase imaging of the filter can directly reflect the local magnetic field inhomogeneity that is caused by the change of the proton spin phase $(10,12)$. Recently, SWI imaging has been applied to mostly study Parkinson's disease (PD), and the increase of iron deposition in the substantia nigra in PD patients $(13,14)$. The magnetic susceptibility of the substantia nigra was likely to change due to the high content of iron. Furthermore, the signal of SWI was low in healthy individuals (15). Therefore, a SWI signal may indicate changes in regional cerebral iron. However, there is not much research on the of correlation between SWI and WD. To the best of our knowledge, the present WD patient who had the Thr935Met/Pro992Leu compound heterozygote mutation in the ATP7B gene is the first to show intra-cerebral paramagnetric signal abnormalities on SWI.

SWI is very sensitive to mineral deposits and has been used as a relatively simple, safe, noninvasive in vitro examination of the deposition of iron metabolism $(10,16)$. Moreover, an SWI scan of the brain is not only used to observe the iron deposition, but also as a sensitive method for quantification $(9,15)$. Abnormal iron deposition is detected before the appearance of clinical symptoms, and it provides information on the pathogenesis $(11,12,16)$. Furthermore, it is an important and novel method for detecting abnormal iron metabolism in a high-risk population $(11,16)$. WD patients are known to have an abnormal copper metabolism but whether they also have iron metabolism abnormalities has not yet been determined (17).

Bruehlmeier et al (18) demonstrates that the uptake of radioactive $52 \mathrm{Fe}$ significantly increased in the pia mater of PD patients from plasma into the brain tissue compared to the 
uptake in healthy individuals. However, the result is not clear and needs further investigation on WD patients with abnormal brain iron metabolism mechanism. In the present case, the lenticular nucleus, substantia nigra and red nucleus have an abnormal SWI signal, and the phase value of corrected phase imaging in WD (Fig. $1 \mathrm{~F}$ and $\mathrm{H}$ ) is more than that of a healthy patient (Fig. 1B and D). The results indicate that in LN/SN/RN there were paramagnetic mineral deposits.

Finally, the case of the present study demonstrates that the SWI hyperintense area lenticular nucleus, substantia nigra and red nucleus in combination with the patient's symptoms indicated a possibility of diagnosing WD when the gene had not been detected. Moreover, it demonstrates that systemic mineral removal treatment, including manganese, iron and copper, may be successful for the primary treatment of WD.

As it is only one case presented here, the conclusions of the present study are limited and further complementary studies involving a larger number of cases are required.

\section{Acknowledgements}

The present study was supported by the Fujian Provincial Health and Family Planning Commission Youth Research Project (grant no. 2015-1-32) and the Key Clinical Specialty Discipline Construction Program of Fujian and Nation in China.

\section{References}

1. Tanzi RE, Petrukhin K, Chernov I, Pellequer JL, Wasco W, Ross B, Romano DM, Parano E, Pavone L, Brzustowicz LM, et al: The Wilson disease gene is a copper transporting ATPase with homology to the Menkes disease gene. Nat Genet 5: 344-350, 1993.

2. La Fontaine S and Mercer JFB: Trafficking of the copperATPases, ATP7A and ATP7B: Role in copper homeostasis. Arch Biochem Biophys 463: 149-167, 2007.

3. La Fontaine S, Ackland ML and Mercer JF: Mammalian coppertransporting P-type ATPases, ATP7A and ATP7B: Emerging roles. Int J Biochem Cell Biol 42: 206-209, 2010
4. Rosencrantz R and Schilsky M: Wilson disease: Pathogenesis and clinical considerations in diagnosis and treatment. Semin Liver Dis 31: 245-259, 2011.

5. Wu F, Wang J, Pu C, Qiao L and Jiang C: Wilson's disease: a comprehensive review of the molecular mechanisms. Int J Mol Sci 16: 6419-6431, 2015.

6. Loudianos G, Lepori MB, Mameli E, Dessì V and Zappu A: Wilson's disease. Pril (Makedon Akad Nauk Umet Odd Med Nauki) 35: 93-98, 2014.

7. Kozic D, Svetel M, Petrovic B, Dragasevic N, Semnic R and Kostic VS: MR imaging of the brain in patients with hepatic form of Wilson's disease. Eur J Neurol 10: 587-592, 2003.

8. Mittal S, Wu Z, Neelavalli J and Haacke EM Susceptibility-weighted imaging: Technical aspects and clinical applications, part 2. AJNR Am J Neuroradiol 30: 232-252, 2009.

9. Haacke EM, Cheng NY, House MJ, Liu Q, Neelavalli J, Ogg RJ, Khan A, Ayaz M, Kirsch W and Obenaus A: Imaging iron stores in the brain using magnetic resonance imaging. Magn Reson Imaging 23: $1-25,2005$.

10. Haacke EM, Xu Y, Cheng YC and Reichenbach JR: Susceptibility weighted imaging (SWI). Magn Reson Med 52: 612-618, 2004.

11. Barnes SR and Haacke EM: Susceptibility-weighted imaging. clinical angiographic applications. Magn Reson Imaging Clin N Am 17: 47-61, 2009.

12. Sehgal V, Delproposto Z, Haacke EM, Tong KA, Wycliffe N, Kido DK, Xu Y, Neelavalli J, Haddar D and Reichenbach JR: Clinical applications of neuroimaging ith susceptibility-weighted imaging. J Magn Reson Imaging 22: 439-450, 2005.

13. Zhang J, Zhang Y, Wang J, Cai P, Luo C, Qian Z, Dai Y and Feng H: Characterizing iron deposition in Parkinson's disease using susceptibility-weighted imaging: An in vivo MR study. Brain Res 1330: 124-130, 2010.

14. Wallis LI, Paley MN, Graham JM, Grünewald RA, Wignall EL, Joy HM and Griffiths PD: MRI assessment of basal ganglia iron deposition in Parkinson's disease. J Magn Reson Imaging 28: 1061-1067, 2008.

15. Haacke EM, Ayaz M, Khan A, Manova ES, Krishnamurthy B, Gollapalli L, Ciulla C, Kim I, Petersen F and Kirsch W: Establishing a baseline phase behavior in magnetic resonance imaging to determine normal vs. abnormal iron content in the brain. J Magn Reson Imaging 26: 256-264, 2007.

16. Haddar D, Haacke E, Sehgal V, Delproposto Z, Salamon G, Seror O and Sellier N. Susceptibility weighted imaging: Theory and applications. J Radiol 85: 1901-1908, 2004.

17. Hingwala DR, Kesavadas C, Thomas B and Kapilamoorthy TR: Susceptibility weighted imaging in the evaluation of movement disorders. Clin Radiol 68: e338-e348, 2013.

18. Bruehlmeier M, Leenders KL, Vontobel P, Calonder C, Antonini A and Weindl A: Increased cerebral iron uptake in Wilson's disease: A 52Fe-citrate PET study. J Nucl Med 41: 781-787, 2000. 